\title{
Production of Dilepton/Photon in Semi-Quark Gluon Plasma
}

\author{
Yoshimasa Hidaka ${ }^{\mathrm{a}}$, Shu Lin ${ }^{\mathrm{b}}$, Robert D. Pisarski ${ }^{\mathrm{b}, \mathrm{c}}$, Daisuke Satow ${ }^{\mathrm{a}, \mathrm{c}}$, Vladimir V. \\ Skokov $^{\mathrm{d}}$ \\ ${ }^{a}$ Theoretical Research Division, Nishina Center, RIKEN, Wako 351-0198, Japan \\ ${ }^{b}$ RIKEN/BNL Research Center, Brookhaven National Laboratory, Upton, NY 11973, USA \\ ${ }^{c}$ Department of Physics, Brookhaven National Laboratory, Upton, NY 11973, USA \\ ${ }^{d}$ Department of Physics, Western Michigan University, 1903 W. Michigan Avenue, Kalamazoo, MI 49008
}

\begin{abstract}
We consider the production of dilepton/photon in deconfined Quark Gluon Plasma (QGP). In the region of semi-QGP, effective color degrees of freedom are suppressed by a small value of the Polyakov loop. We find that the effect of the Polyakov loop leads to modest enhancement in the dilepton production rate but strong suppression in the photon production rate. The suppression of photon production in the QGP phase offers a possible explanation to the puzzle of photon elliptic flow.
\end{abstract}

Keywords: Semi-QGP, Polyakov loop, Electromagnetic radiation, Elliptic flow

\section{Introduction}

It is believed that a deconfined QGP is produced at both Relativistic Heavy Ion Collider (RHIC) and Large Hadron Collider (LHC). The properties of the QGP and its evolution have been under intensive theoretical and experimental study. Electromagnetic radiation, i.e. dilepton/photon production is of particular interest among multiple experimental probes of the QGP. Once produced, electromagnetic radiation escapes from the QGP without further interaction, thus it provides a clear probe of spacetime evolution of the QGP. Since the radiation is also produced from other sources, including the hadronic phase and initial hard scattering, it is desirable to pin down the radiation in the QGP phase theoretically.

This is a difficult task: While perturbative field theory is applicable at high temperature, QGP produced both at RHIC and LHC spends most of its life time in the temperature window $1 \sim 2 T_{c}$, with $T_{c}$ being the deconfinement temperature. This is coined the semi-QGP region [1]. Lattice simulation shows that this region is characterized by a smooth rise of the Polyakov loop [2], an effect missed by perturbative field theory. In models of the semi-QGP, the nontrivial value of Polyakov loop can be modeled by mean field theory. With the mean field as background, we perform a calculation of dilepton/photon production in the semi-QGP. We find stark difference between the two: the dilepton production is modestly enhanced, while the photon production is strongly suppressed. In the region with negligible prompt photon contribution, the suppressed photon rate in the QGP phase implies that more photons are produced in the hadronic phase. Since the hadronic phase has built up sufficient flow in the evolution of the QGP, our result offers a possible explanation to the puzzle of photon elliptic flow $v_{2}$ [3]. 


\section{Semi-QGP and effect on electromagnetic radiation}

The Polyakov loop is defined as the trace of a Wilson loop wrapped around the compactified time circle

$$
l=\frac{1}{N_{c}} \operatorname{tr} \mathcal{P} \exp \left(i g \int_{0}^{1 / T} d \tau A_{0}\right),
$$

where $g$ is the gauge coupling and $A_{0}$ the time component of the gluon field in Euclidean space. Lattice simulation shows that the expectation value $\langle l\rangle$ is small in the confined phase, and approaches unity at high temperature. In the semi-QGP region, $\langle l\rangle$ interpolates between a small value to a modest one. The Polyakov loop can be modeled by a constant background field $A_{0}$ with diagonal entries $Q_{a} / g\left(a=1,2, \ldots, N_{c}\right)$ only. This gives a Polyakov loop $l=1 / N_{c} \sum_{a} e^{i Q_{a} / T}$. For three colors, we can choose $A_{0}=(Q, 0,-Q) / g$. The Polyakov loop is then expressed as

$$
l=\frac{1}{3}\left(1+2 \cos \frac{Q}{T}\right)
$$

The value of the color charge $Q$ at a given temperature can be determined from lattice data of the Polyakov loop $l$, with the perturbative contribution properly removed [4]. Under Wick rotation, the background charge is purely imaginary in Minkowski space. The presence of the background color charge serves as imaginary chemical potential. For quarks with color index $a$, the energy is modified as $p^{0} \rightarrow p^{0}-i Q_{a}$; for gluons with color indices $a, b$, the energy is modified as $p^{0} \rightarrow p^{0}-i Q_{a}+i Q_{b}$. The imaginary energy is an effective way of describing the influence of the Polyakov loop on quarks and gluons. Physical quantities are obtained after summing over color indices. In particular, three of the authors found that the Polyakov loop generically suppresses quark and gluon number, consequently they found a reduced energy loss for a heavy quark traveling in the semi-QGP [4]. Since the basic processes of dilepton/photon production also involve taking color objects from the QGP medium, does this imply a reduced dilepton/photon rate?

We first consider the case of dilepton production. The leading order rate is given by the fusion of a quark/anti-quark pair into a virtual photon. The standard perturbation theory gives

$$
\left.\frac{d \Gamma}{d^{4} P}\right|_{Q=0}=\frac{\alpha_{e m}}{6 \pi^{4}} n(E)\left(1-\frac{2 T}{p} \ln \frac{N\left(p_{-}\right)}{N\left(p_{+}\right)}\right),
$$

where $n(E)=1 /\left(e^{E / T}-1\right)$ is the Bose-Einstein distribution, and $N\left(p_{ \pm}\right)=1+e^{-p_{ \pm} / T}$ is related to the Fermi-Dirac distribution, with $p_{ \pm}=\frac{E \pm|\vec{p}|}{2}$. We have assumed three flavors of massless quarks in the medium. With background color charge, the dilepton rate is modified to

$$
\left.\frac{d \Gamma}{d^{4} P}\right|_{Q \neq 0}=\left.\frac{\tilde{f}_{l \bar{l}}(Q)}{\tilde{f}_{l \bar{l}}(0)} \frac{d \Gamma}{d^{4} P}\right|_{Q=0},
$$

where $\tilde{f}_{l \bar{l}}(Q)$ is expressed in terms of the Polyakov loop

$$
\tilde{f}_{i \bar{l}}(Q)=1-\frac{2 T}{3 p} \ln \frac{1+3 l e^{-p_{-} / T}+3 l e^{-2 p_{-} / T}+e^{-3 p_{-} / T}}{1+3 l e^{-p_{+} / T}+3 l e^{-2 p_{+} / T}+e^{-3 p_{+} / T}} .
$$

In the special case where the dilepton is produced back to back, $p=0$, we plot in Fig. 1 the modification factor $f_{l \bar{l}}(Q)=\frac{\tilde{f}_{\bar{l}}(Q)}{\tilde{f}_{\bar{l}}(0)}$ at $E=1 \mathrm{GeV}$, as a function of temperature. We find that $f_{l \bar{l}}(Q)$ is always greater than unity. The non-suppression of the dilepton rate can be understood as follows: The quark and anti-quark producing a virtual photon carry opposite imaginary chemical potential, with their distribution functions given by $n_{a}=\frac{1}{e^{(E / 2-i Q a) / T}+1}$ and $\bar{n}_{a}=\frac{1}{e^{(E / 2+i Q a) / T}+1}$. For high energy dilepton $E \gg T$, we can approximate the distribution function by a Boltzmann factor, which gives the following rate for dilepton production

$$
e^{2} \sum_{a=1}^{N_{C}} e^{-\left(E / 2-i Q_{a}\right) / T} e^{-\left(E / 2+i Q_{a}\right) / T}\left|\mathcal{M}_{l \bar{l}}\right|^{2}
$$

where $\left|\mathcal{M}_{\bar{l}}\right|^{2}$ is the square of the amplitude for virtual photon production from a quark/anti-quark pair. We see that although the individual quark/anti-quark is suppressed in the semi-QGP [4], their product has no suppression due 


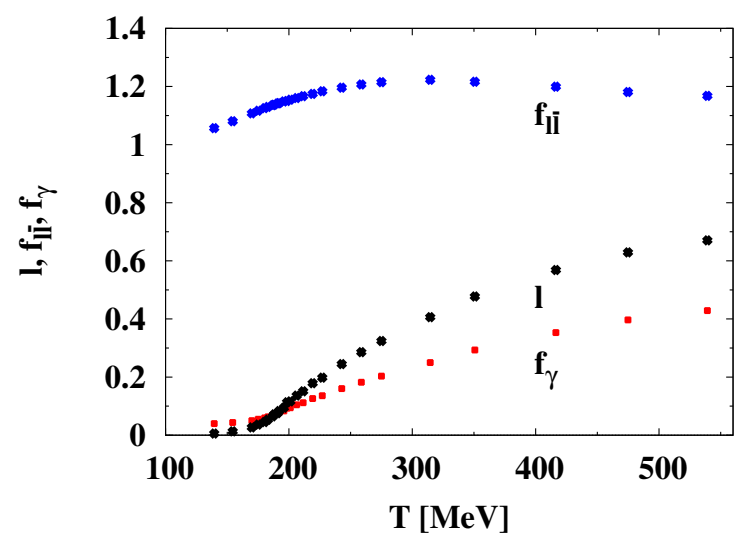

Figure 1. The modification factors due to background color charge as a function of temperature, for dilepton production $f_{l \bar{l}}$ at energy $E=1 \mathrm{GeV}$ and $p=0$, and for photon production $f_{\gamma}$, which is independent of photon energy. The Polyakov loop as a function of temperature is also included in the plot.

to the cancellation between the opposite chemical potentials. At modestly high energy, the product is even slightly enhanced, as is clear from Fig.1.

The effect of background color charge on the photon rate is very different. The dominant processes contributing to the photon rate are the $2 \rightarrow 2$ processes including Compton scattering and pair annihilation. In the semi-QGP, the rate at leading logarithmic order factorizes

$$
\left.E \frac{d \Gamma}{d^{3} p}\right|_{Q \neq 0}=\left.f_{\gamma}(Q) E \frac{d \Gamma}{d^{3} p}\right|_{Q=0},
$$

with the standard leading logarithmic rate from $2 \rightarrow 2$ processes [5]

$$
\left.E \frac{d \Gamma}{d^{3} p}\right|_{Q=0}=\frac{\alpha_{e m} \alpha_{s}}{3 \pi^{2}} e^{-E / T} T^{2} \ln \frac{E}{g^{2} T},
$$

and the energy independent modification factor

$$
f_{\gamma}(Q)=1-4 q+\frac{10}{3} q^{2} ; \quad q=\frac{Q}{2 \pi T}, \quad 0<q<1 .
$$

We include the modification factor in Fig.1, together with the Polyakov loop itself. We observe a strong suppression for photon production. Since both photon and dilepton represent color neutral excitation in the medium, why do they show such different behavior? To understand this, we need to take a close look at the $2 \rightarrow 2$ processes. Let us first consider pair annihilation. In this case quark and anti-quark in the initial state do not have to carry the same color. This is because a gluon also appears in the final state in addition to the photon. Similar to the case of dilepton production, we use the Boltzmann approximation for the incoming quark/anti-quark, and approximate the Bose-enhancement factor for the gluon by one to obtain the following rate:

$$
e^{2} g^{2} \sum_{a, b=1}^{N_{c}} e^{-\left(E_{1}-i Q_{a}\right) / T} e^{-\left(E_{2}-i Q_{b}\right) / T}\left|\mathcal{M}_{\gamma}^{a b}\right|^{2},
$$

where $\left|\mathcal{M}_{\gamma}^{a b}\right|^{2}$ is the square of the amplitude for pair annihilation. Now that the color indices do not match, each color sum contributes a factor of a Polyakov loop $\sum_{a=1}^{N_{c}} e^{-i Q_{a} / T}=N_{c} l$. The analysis of Compton scattering proceeds analogously. In that case, the color index of an incoming quark matches with one of the color indices of an incoming gluon, leaving one unmatched color index, thereby the rate of Compton scattering is suppressed by only one factor of the Polyakov loop in that approximation. 
The above discussion has ignored one contribution to photon production, which is known as the collinear rate in standard thermal field theory. We now argue it is parametrically suppressed with respect to $2 \rightarrow 2$ processes. We recall that the collinear rate comes from the radiation from a quark undergoing multiple scatterings with soft gluons, which requires summation of infinitely many diagrams [6]. In order for these processes to contribute, it is important that the gluons are soft $E_{g} \sim g T$, such that their number density is Bose-Einstein enhanced $n\left(E_{g}\right) \sim \frac{1}{g}$ to compensate for the additional $g$ from the quark-gluon vertex. In the semi-QGP, the energy of the gluon should be modified as $E_{g} \rightarrow E_{g}+i Q_{a}-i Q_{b}$. Because $Q_{a}-Q_{b} \sim T$ for $a \neq b$, the enhancement is not present for off-diagonal gluons. Only diagonal gluons with $a=b$ can contribute in these processes. This introduces a factor of $1 / N_{c}$, in addition to factors of the Polyakov loop from quark distribution functions, for the collinear rate in the semi-QGP. Therefore, we ignore the collinear rate in our discussion.

\section{Discussion}

We have calculated dilepton and photon production in the semi-QGP. We find a modest enhancement for the dilepton rate and a strong suppression for the photon rate. The difference can be understood in terms of the initial states: A color neutral initial state does not lead to suppression; a colored initial state generically leads to suppression by the Polyakov loop.

There are three main sources of direct photon production: QGP phase, hadronic phase and initial hard scattering (prompt photon). The prompt photon dominates for high energy photons, while QGP and hadronic phases provide the main contributions for photons with modest energy and below. Our results indicate a strong suppression of photon production in the QGP phase, which increases the relative weight of photon production in the hadronic phase. This favors a large elliptic flow for photons, thus offering a possible explanation to the puzzle of photon $v_{2}$. On the other hand, our results also show a modest enhancement for dilepton in the QGP phase, which implies that dilepton $v_{2}$ might not be as significant as that of the photon. To answer the questions in a quantitative level it is necessary to include dilepton/photon production from all sources and convolute them with hydrodynamic evolution of the fireball [7].

\section{Acknowledgments}

S.L. is supported by the RIKEN Foreign Postdoctoral Researchers Program. R.D.P is supported by the U.S. Department of Energy under contract \#DE-AC02-98CH10886. D.S. is supported by JSPS Strategic Young Researcher Overseas Visits Program for Accelerating Brain Circulation (No. R2411).

\section{References}

[1] A. Dumitru, Y. Guo, Y. Hidaka, C. P. K. Altes and R. D. Pisarski, Phys. Rev. D 83, 034022 (2011) [arXiv:1011.3820 [hep-ph]]; A. Dumitru, Y. Guo, Y. Hidaka, C. P. K. Altes and R. D. Pisarski, Phys. Rev. D 86, 105017 (2012) [arXiv:1205.0137 [hep-ph]].

[2] A. Bazavov, T. Bhattacharya, M. Cheng, N. H. Christ, C. DeTar, S. Ejiri, S. Gottlieb and R. Gupta et al., Phys. Rev. D 80, 014504 (2009) [arXiv:0903.4379 [hep-lat]].

[3] A. Adare et al. [PHENIX Collaboration], Phys. Rev. Lett. 109, 122302 (2012) [arXiv:1105.4126 [nucl-ex]].

[4] S. Lin, R. D. Pisarski and V. V. Skokov, Phys. Lett. B 730, 236 (2014) [arXiv:1312.3340 [hep-ph]].

[5] R. Baier, H. Nakkagawa, A. Niegawa and K. Redlich, Z. Phys. C 53, 433 (1992); J. I. Kapusta, P. Lichard and D. Seibert, Phys. Rev. D 44, 2774 (1991) [Erratum-ibid. D 47, 4171 (1993)].

[6] P. Aurenche, F. Gelis and H. Zaraket, Phys. Rev. D 62, 096012 (2000) [hep-ph/0003326]; P. B. Arnold, G. D. Moore and L. G. Yaffe, JHEP 0206, 030 (2002) [hep-ph/0204343]; P. B. Arnold, G. D. Moore and L. G. Yaffe, JHEP 0112, 009 (2001) [hep-ph/0111107].

[7] C. Gale, Y. Hidaka, S. Jeon, S. Lin, J.-F. Paquet, R. D. Pisarski, D. Satow, V. V. Skokov and G. Vujanovic, arXiv:1409.4778 [hep-ph]. 